\title{
Monte Carlo technique with a quantified time step: Application to the motion of magnetic moments
}

\author{
O. Chubykalo \\ Instituto de Ciencia de Materiales de Madrid, CSIC, Cantoblanco, E-28049 Madrid, Spain \\ U. Nowak \\ Theoretische Tieftemperaturphysik, Gerhard-Mercator-Universität-Duisburg, D-47048 Duisburg, Germany \\ R. Smirnov-Rueda \\ Departamento de Matematica Aplicada, Facultad de Ciencias Matematicas, Universidad Complutense de Madrid, 28040 Madrid, Spain \\ M. A. Wongsam \\ Department of Physics, University of Durham, South Road, Durham, D1 3LE, United Kingdom \\ R. W. Chantrell \\ Seagate Research, River Parks Commons, 2403 Sydney Street, Pittsburgh, Pennsylvania 15203-2116 \\ J. M. Gonzalez \\ Instituto de Ciencia de Materiales de Madrid, CSIC, Cantoblanco, E-28049 Madrid, Spain \\ and Instituto de Magnetismo Aplicado, RENFE/UCM, Las Rozas, Madrid, Spain
}

(Received 21 March 2002; published 28 February 2003)

\begin{abstract}
The viability of the time quantified Metropolis Monte Carlo technique to describe the dynamics of magnetic systems is discussed. Similar to standard Brownian motion, the method is introduced basing on the comparison between the Monte Carlo trial step and the mean squared deviation of the direction of the magnetic moment. The Brownian dynamics approach to the time evolution of a magnetic moment is investigated and expressions for the mean square deviations are obtained. However, the principle difference between the standard Brownian motion and the magnetic moments dynamics is the presence of the spin precession which constitutes the reversible part of the dynamics. Although some part of the precession contributes to the diffusion coefficient, it also gives rise to athermal, energy conserving motion which cannot be taken into account by Monte Carlo methods. It is found that the stochastic motion of a magnetic moment falls into one of two possible regimes: (i) precession dominated motion, (ii) nonprecessional motion, according to the value of the damping constant and anisotropy strength and orientation. Simple expressions for the diffusion coefficient can be obtained in both cases for diffusion dominated motion, i.e., where the athermal precessional contribution can be neglected. These simple expressions are used to convert the Monte Carlo steps to real time units. The switching time for magnetic particles obtained by the Monte Carlo with time quantification is compared with the numerical integration of the Landau-Lifshitz-Gilbert equations with thermal field contribution and with some well known asymptotic formulas.
\end{abstract}

DOI: 10.1103/PhysRevB.67.064422

PACS number(s): 75.40.Gb, 75.40.Mg, 75.50.Tt

\section{INTRODUCTION}

The problem of thermally induced magnetization reversal is very important from both, a fundamental and an applied point of view. In magnetic recording applications recently this problem has become of particularly keen interest since it is widely accepted that the superparamagnetic recording density limit will shortly be achieved. Several methods of treating the problem in different time scales exist already in the literature.

On time scales less than $1 \mathrm{~ns}$, one normally integrates the dynamical equation of motion (Landau-Lifshitz-Gilbert) with the thermal field representing thermal fluctuations following Brown. ${ }^{1}$ On large time scales up to the order of years, the kinetic Monte Carlo (MC) method ${ }^{2}$ which is known in micromagnetics as the Charap method ${ }^{3}$ can be used. This statistical method is equivalent to the solution of the two-level Master equation. ${ }^{2,4}$ The method supposes that the system could be found only in the energy minima and calculates the energy barriers separating them, assuming the ArrheniusNéel law for the probability of transition. As a consequence, the method could be viable for barriers large enough to build the equilibrium statistics. The dynamical information is contained in the Arrhenius-Néel prefactor. This constant is normally calculated using the Fokker-Planck equation ${ }^{5-8}$ and is known exactly only for a few simple cases.

At intermediate time scales, say, up to $1 \mathrm{~s}$, the correct precessional information may not be important. However, some dynamical information arising from the form of the potential in which the particle is moving may still be necessary. The time quantified Metropolis Monte Carlo (TQMC) algorithm ${ }^{9,10}$ has been designed to work in this intermediate time scale regime. It has been applied, for example, to calculate the magnetization decay in a di-bit pattern as a function of exchange parameter and gave a similar result in comparison to other methods. ${ }^{11}$ Consequently, it is very 
important to compare the integration of the Landau-Lifshitz equation and the statistical (analytical asymptote) approaches with the TQMC method where their respective time regimes overlap.

Metropolis MC methods are well established in the context of equilibrium thermodynamics. ${ }^{12,13}$ However, Metropolis $\mathrm{MC}$ is also viable to describe nonequilibrium dynamics. The most common example is a random walk of a Newtonian particle in the presence of thermal fluctuations. It is well known ${ }^{14}$ that the dynamics of a Brownian particle (here we consider as Brownian particle a Newtonian particle in an external potential with a random term added to the equation of motion to simulate the temperature) obeys a simple diffusion equation. For a small time step the Master equation which governs the MC procedure is equivalent to the Fokker-Planck equation. ${ }^{15}$ Kikuchi et al. ${ }^{16}$ showed directly that for a Brownian particle in an external potential the Metropolis MC could be viewed as a numerical method to solve the corresponding Fokker-Planck equation. Although this was not stated in the paper, but it may be deduced that if the fluctuation sizes within a MC step are chosen to match the diffusion property of a random walk, i.e.,

$$
\left\langle x^{2}\right\rangle=2 D \Delta t,
$$

where $D$ is the diffusion coefficient, the Metropolis MC procedure should adequately describe the dynamics. The comparison of the nonequilibrium probability functions obtained by the MC and the analytical solution was in complete agreement. Meiburg ${ }^{17}$ also presented in his paper direct comparison of the simulation of the Reyleigh-Stokes flow obtained by the $\mathrm{MC}$ and the molecular dynamics method.

In magnetism, mainly Ising and Heisenberg models have been investigated by MC to account for equilibrium properties (critical phenomenon) due to the broad variety of applications of this class of models in statistical physics. For nonequilibrium dynamics, the MC method was used by Gonzalez et al. ${ }^{18-21}$ to account for thermally induced collective magnetization relaxation. It has been shown, for example, that MC produces the expected logarithmic time dependence of magnetization after some waiting time.

Therefore, Metropolis MC has recommended itself in magnetism as a fast computational method producing physically reasonable dynamics. The big disadvantage of the MC algorithm is that the time step is normally measured in $\mathrm{MC}$ steps and the physical time involved in the calculations is unknown. The connection to physical time-if there is one at all-is in general an open problem which is settled up to now only in certain very simple cases. For example, for a Brownian motion, if we are able to present some simple formula for the diffusion coefficient (1.1) calculated from the dynamical equation and use it to quantify the time step corresponding to a MC trial step, then the MC procedure should correctly describe the statistical properties of the time evolution of the system. Of course, the main difference between a simple Brownian particle and a magnetic moment is the presence of the precessional term. This difference is crucial and does not allow mere translation of the results from one system to another. Generally speaking, a Metropolis MC simulation (in a canonical ensemble) simply does not include the energy con- serving part of the equation of motion. Hence, no spin precession scenario can be simulated by means of this approach. On the other hand, the random-walk-like motion which is due to the coupling of the system of interest to the heat bath is paramount. As a consequence, the MC procedure will work for a magnetic system dynamics where and when its dynamics is similar to that of a simple random walk.

The work of Nowak et al. ${ }^{9}$ introduced the so-called Monte Carlo method with quantified time step by comparing the fluctuation size produced by MC to that produced by the Langevin dynamics. However, it was assumed that the magnetization fluctuations have the same simple time dependence as the thermal force fluctuations by analogy to the results for the Brownian particle. For magnetic systems this idea is not straightforward due to the presence of precession and in the present paper we calculate and analyze the diffusion coefficient (1.1) directly from the linearized equation of motion for magnetic moments. We also try to explain how far a TQMC procedure can in general be applied for the investigation of a dynamical behavior and we establish in detail the necessary conditions for its application. Unlike previous comparisons between $\mathrm{MC}$ and Langevin dynamics or analytical calculations ${ }^{16,17}$ we present our results in real time units corresponding to average switching time of magnetic particles which we choose as an example of essentially nonequilibrium dynamics. As far as we know, this has never been done before. The main idea of the present paper is to work further on the method which is capable to calculate the switching processes of magnetic structures in the presence of temperature and is fast comparing to that of the molecular dynamics.

\section{DIFFUSIONAL PROPERTIES OF A SINGLE MAGNETIC MOMENT IN A POTENTIAL WELL}

As was mentioned above, the existence of the precessional motion makes the magnetic moment dynamics different from that of the Brownian particle. From the point of view of the $\mathrm{MC}$ technique, it is important to find out under which conditions the statistical properties of the two dynamics are similar and to calculate the diffusion coefficient (1.1) which could be used in the time quantification. Let us start with some simple observations of the magnetic particle motion governed by the Landau-Lifshitz-Gilbert equation with a random field representing thermal fluctuations. The equation is written in the following form:

$$
\frac{d \vec{M}}{d \tau}=-\vec{M} \times \vec{H}-\alpha \vec{M} \times[\vec{M} \times \vec{H}],
$$

where

$$
\tau=\frac{\gamma_{0} H_{k}}{M_{s}\left(1+\alpha^{2}\right)} t
$$

$\gamma_{0}$ is the gyromagnetic ratio, and $\alpha$ is the damping constant. The magnetic moment $\vec{M}$ is normalized to the saturation value $M_{s}$, and the internal field $\vec{H}$, given by 


$$
\vec{H}=-\frac{\delta E^{*}}{\delta \vec{M}},
$$

is normalized to the anisotropy field $H_{k}=2 K / M_{s}$ where $K$ is the anisotropy value. The energy $E^{*}=E / 2 K V$, where $V$ is the volume, contains all the necessary energy contributions: anisotropy, exchange, magnetostatic, and Zeeman. The thermal fluctuations are introduced as random field fluctuations, added to a total internal field (2.3) and having statistical properties given by

$$
\begin{gathered}
\left\langle\xi_{i}\right\rangle=0, \\
\left\langle\xi_{i}(0) \xi_{j}(\tau)\right\rangle=\frac{\alpha k_{B} T}{K V\left(1+\alpha^{2}\right)} \delta_{i j} \delta(\tau),
\end{gathered}
$$

where $i, j$ denote Cartesian components $x, y, z$. In what follows we will compare the results of the MC simulations with the integration of the Landau-Lifshitz-Gilbert equation. The majority of the results of this paper are related to one moment, or an assembly of noninteracting moments, with a given anisotropy easy axis and external field $H_{\text {app }}$ applied at some angle to it. In this case the $z$ axis is assumed to be parallel to the equilibrium magnetic moment direction and the $x$ axis to be in the plane containing the equilibrium direction defined by the anisotropy axis and the external field direction, and the $y$ axis is perpendicular to this plane. The total energy can be written as

$$
E^{*}=-\frac{1}{2}\left(M_{x} n_{x}+M_{z} n_{z}\right)^{2}-\vec{M} \cdot \vec{H}_{\mathrm{app}},
$$

where $M_{x}, M_{z}$ and $n_{x}, n_{z}$ are projections of the magnetic moment $\vec{M}$ and the easy axis unit vector $\vec{n}$ on the $x, z$ axes, respectively. In the Sec. IV of the present paper other examples will be considered.

\section{A. General observations on the stochastic motion of the magnetic moment close to the potential minimum}

Let us consider a magnetic moment with initial condition at the equilibrium position. If thermal fluctuations were absent, no torques would exist and the moment would remain at the zero temperature mechanical equilibrium. However, the thermal field leads to the establishment of a finitetemperature thermal equilibrium which is meaningful only in a statistical sense. Figure 1 presents the mean squared deviation of the moment's $M_{x}$ and $M_{y}$ components from their equilibrium values averaged over many realizations. It can be noticed that initially the mean squared deviation is linear in time and could be viewed as the motion of a simple, free Brownian particle. At longer times, the mean squared deviations achieve their thermal equilibrium values which should correspond to the stationary solutions of the Fokker-Planck equation. ${ }^{5-8}$ Note that these stationary values are different for $x$ and $y$ components.

The existence of these equilibrium values does not mean that the moment performs a precessional motion (with some thermal perturbation) around some equilibrium stable cycles. In what follows we will consider a particle in a so-called

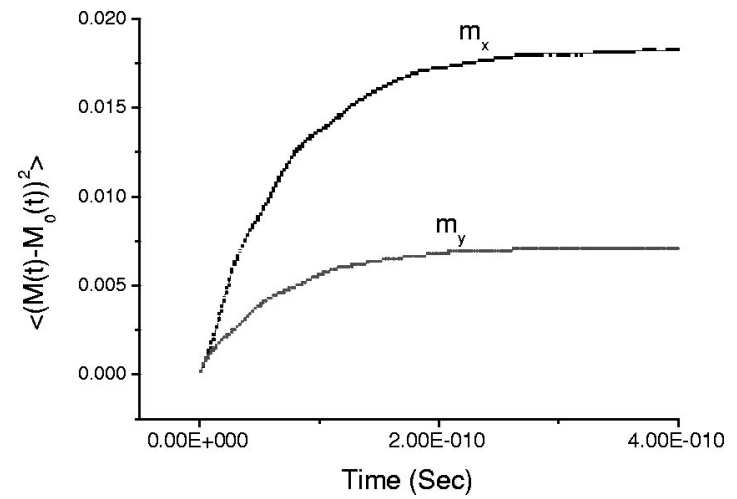

FIG. 1. Temporal evolution of the mean squared deviation from equilibrium. Average over 10000 trajectories. $K V / k_{B} T=4.16, \alpha$ $=0.1$. Angle between the applied field and anisotropy axis is $\pi / 2$.

statistical regime, i.e., when the time spent by a particle in a potential well is enough to build an equilibrium statistics (large barrier case). In Fig. 2 we present the equilibrium distribution, that is, excluding the initial dynamical part, of the $M_{y}$ component for a fixed value of the $M_{x}$ component for the cases of large and small damping. We see that in both cases the particle mean position is in the nonthermal (i.e., mechanical) equilibrium. As a consequence, the moment spends the majority of time near the nonthermal equilibrium position corresponding to intervals $M_{x, y, z}^{0} \pm \sqrt{\left\langle\left(\Delta M_{x, y, z}\right)^{2}\right\rangle}$. This is in the spirit of the solution of the Fokker-Planck equation $^{5-8}$ which assumes the equilibrium Boltzmann distribution even in the small damping case. Note that in the case presented in Fig. 2 the equilibrium distributions are independent of the value of the damping constant. If the temperature is not high, it is reasonable to assume that for most of the time the magnetic motion will satisfy the condition $\Delta M_{x, y, z} \ll 1$. In this case the linear approximation can be used and the corresponding stochastic equations of motion can be solved exactly. The time corresponding to the large magnetization deviation from the equilibrium will constitute a small part of the total escape time from one equilibrium position to another.

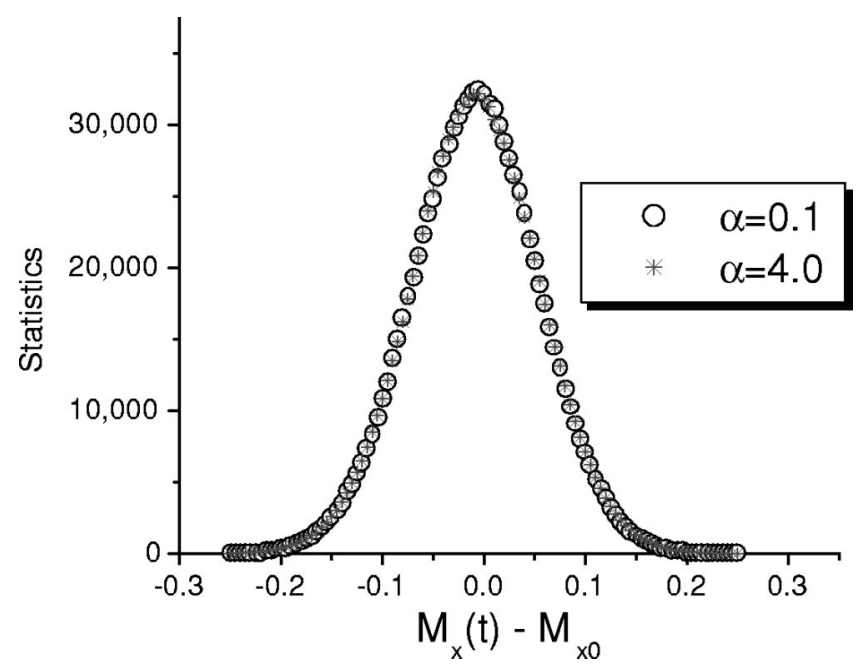

FIG. 2. Distribution of moment deviations of the $x$ component for fixed $y$ component, for two values of $\alpha . K V / k_{B} T=7.18$. Angle between the applied field $H=0.9 K_{z} V$ and anisotropy axis is $\pi / 2$. 


\section{B. Linearized equation of motion for a single magnetic moment}

It has been shown that once the temporal evolution of the second moments are known, then so are the diffusion coefficients and in principle, the statistical properties for the time evolution of the Brownian particle can be investigated by TQMC. For a magnetic moment, in a general case no closed equations exist. ${ }^{22}$ However, in many cases the linear approximation may be sufficient. We linearize the dynamical equation of motion for one magnetic moment with energy given by Eq. (2.6). Since the length of the magnetization vector is a constant, this energy expression should contain an additional term $\frac{1}{2} \lambda_{L} M^{2}$, where $\lambda_{L}$ is the Lagrange multiplier.

The corresponding energy gradients are related to the effective field values used in the Landau-Lifshitz-Gilbert equation

$$
\begin{gathered}
H_{x}^{\mathrm{eff}}=\left(M_{x} n_{x}+M_{z} n_{z}\right) n_{x}+H_{x}-\lambda_{L} M_{x}, \\
H_{y}^{\mathrm{eff}}=-\lambda_{L} M_{y}, \\
H_{z}^{\mathrm{eff}}=\left(M_{x} n_{x}+M_{z} n_{z}\right) n_{z}+H_{z}-\lambda_{L} M_{z} .
\end{gathered}
$$

Taking into account that in the equilibrium state it is $M_{x 0}$ $=M_{y 0}=0$ and $M_{z 0}=1$, we can linearize these expressions in terms of small deviations $m_{x}, m_{y}$, and $m_{z}$ from the equilibrium configuration. As a result, the linearized LandauLifshitz-Gilbert equation for $x$ and $y$ components will have the form

$$
\begin{gathered}
\frac{d m_{x}}{d \tau}=A m_{y}-\alpha B m_{x}, \\
\frac{d m_{y}}{d \tau}=-B m_{x}-\alpha A m_{y},
\end{gathered}
$$

where $A=n_{z}^{2}+H_{0 z}$ and $B=A-n_{x}^{2}$ and $H_{0 z}$ is the equilibrium internal field.

Assuming that the angle between the equilibrium direction and the anisotropy easy axis is $\Theta_{0}$ one can express the coefficients $A$ and $B$ as

$$
\begin{gathered}
A=\cos ^{2} \Theta_{0}-H_{\mathrm{app}} \cos \left(\Theta_{0}+\varphi\right), \\
B=A-\sin ^{2} \Theta_{0},
\end{gathered}
$$

where $\varphi$ is the angle between the negative $z$ axis and applied field.

The thermal fluctuations are introduced to the system according to the fluctuation-dissipation theorem. ${ }^{23}$ Finally we obtain the following linearized Landau-Lifshitz-Gilbert (LLG) equation with additive noise terms

$$
\begin{gathered}
\frac{d m_{x}}{d \tau}=A m_{y}-\alpha B m_{x}+f_{x}, \\
\frac{d m_{y}}{d \tau}=-B m_{x}-\alpha A m_{y}+f_{y},
\end{gathered}
$$

where the random fluctuations possess the following statistics:

$$
\left\langle f_{k}\right\rangle=0 ; \quad\left\langle f_{i}(\tau) f_{j}(s)\right\rangle=\sigma^{2} \delta_{i j} \delta(\tau-s) ; \quad \sigma^{2}=\frac{\alpha k_{B} T}{K V} .
$$

In Eqs. (2.14) and (2.15), $m_{x}$ and $m_{y}$ represent small magnetization fluctuations around the equilibrium values due to the white noise torques $f_{k}$ or due to white noise field $\xi_{k}$. In this particular system of coordinates the two noise representations lead to the same equations.

We first solve the nonstochastic homogeneous equations (2.10) and (2.11) using a trial solution $m_{i}=e^{\lambda t}$ and obtain the eigenvalues

$$
\lambda_{1,2}=-\frac{\alpha(A+B)}{2} \pm \sqrt{\alpha^{2}\left(\frac{A-B}{2}\right)^{2}-A B} .
$$

If $\alpha>\alpha_{\text {cr }}$, where

$$
\alpha_{\mathrm{cr}}=\frac{2 \sqrt{A B}}{|A-B|}
$$

the eigenvalue is real and is given by

$$
\lambda_{1,2}=\frac{-\alpha(A+B)}{2} \pm \omega
$$

where $\omega=\sqrt{\alpha^{2}[(A-B) / 2]^{2}-A B}$. Otherwise, it is complex and given by

$$
\lambda_{1,2}=\frac{-\alpha(A+B)}{2} \pm i \omega
$$

where $\omega=\sqrt{\left.A B-\alpha^{2}[(A-B) / 2)\right]^{2}}$. Note that $\omega$ defines the precession frequency and depends on the damping parameter $\alpha$. The second case is the most typical one, since the condition $|A-B| \ll A, B$, in our case $\sin ^{2} \Theta_{0} \ll 1$, is easily fulfilled, especially in the case of strong anisotropy systems (relatively large barriers).

We now discuss the two regimes determined by the reality/complexity of the eigenvalues, which we designate as precessional or nonprecessional according to whether the eigenvalues are complex or real. Our main goal is to find analytically the regimes where the diffusion coefficient of a magnetic particle is so simple as that of a Brownian particle so that it can be implemented in TQMC.

\section{Precessional case}

In the case of complex eigenvalues we find the solution

$$
\begin{aligned}
m_{x}= & e^{-\alpha^{\prime} \tau}\left\{C_{1}^{0} \cos \omega \tau+C_{2}^{0} \sin \omega \tau\right\}+e^{-\alpha^{\prime} \tau}\left\{C_{1}(\tau) \cos \omega \tau\right. \\
& \left.+C_{2}(\tau) \sin \omega \tau\right\} \\
m_{y}= & e^{-\alpha^{\prime} \tau}\left\{\widetilde{C}_{1}^{0} \cos \omega \tau+\widetilde{C}_{2}^{0} \sin \omega \tau\right\}+e^{-\alpha^{\prime} \tau}\left\{\widetilde{C}_{1}(\tau) \cos \omega \tau\right. \\
& \left.+\widetilde{C}_{2}(\tau) \sin \omega \tau\right\}
\end{aligned}
$$

where $\alpha^{\prime}=\alpha(A+B) / 2$ and 


$$
\begin{aligned}
& \widetilde{C}_{1}=\frac{(B-A) \alpha}{2 A} C_{1}+\frac{\omega}{A} C_{2}, \\
& \widetilde{C}_{2}=\frac{(B-A) \alpha}{2 A} C_{2}-\frac{\omega}{A} C_{1} .
\end{aligned}
$$

The resulting two linear first-order ordinary differential equations for $C_{1,2}(\tau)$ and $\widetilde{C}_{1,2}(\tau)$, corresponding to the solution of the nonhomogeneous equation, can be formally integrated with the initial conditions $m_{x}(\tau=0)=m_{x}^{0}, m_{y}(\tau$ $=0)=m_{y}^{0}$. In what follows we are interested in the diffusion coefficient

$$
\left\langle\left(m_{x}-m_{x}^{0}\right)^{2}\right\rangle=\left(m_{x}-m_{x}^{0}\right)_{\text {atherm }}^{2}+\left\langle\left(m_{x}-m_{x}^{0}\right)^{2}\right\rangle_{\text {therm }},
$$

where the first term comes from the solution of the homogeneous equation and describes the purely athermal precessional motion while the second term includes thermal effects and configurational averaging.

The first term contains the elliptical motion and for short times, $\left(\alpha^{\prime} \tau \ll 1, \omega \tau \ll 1\right)$ we find that it has a quadratic dependence

$$
\left(m_{x}-m_{x}^{0}\right)^{2} \sim A m_{y}^{0} \tau^{2} .
$$

For the second term we obtain

$$
\begin{aligned}
\left\langle\left(m_{x}-m_{x}^{0}\right)^{2}\right\rangle_{\text {therm }}= & \sigma^{2}\left(\frac{1}{2 \alpha B}+e^{-2 \alpha^{\prime} \tau}\left\{\frac{1}{2 A B\left(1+\alpha^{2}\right)}\right.\right. \\
& \times\left[\left(\rho \omega+\frac{B-A}{2 \omega} \alpha \alpha^{\prime}\right) \sin \omega \tau\right. \\
& \left.\left.\left.+\left(\frac{B-A}{2} \alpha-\rho \alpha^{\prime}\right) \cos 2 \omega \tau\right]-\frac{A}{2 \omega^{2} \alpha}\right\}\right),
\end{aligned}
$$

where

$$
\rho=\frac{1}{2}\left\{1-\left(\frac{A}{\omega}\right)^{2}-\left(\frac{(A-B) \alpha}{2 \omega}\right)^{2}\right\}
$$

with a similar equation for $\left\langle\left(m_{y}-m_{y}^{0}\right)^{2}\right\rangle_{\text {therm }}$. For the spatial correlation function we get

$$
\begin{aligned}
\left\langle m_{x} m_{y}\right\rangle_{\text {therm }}= & \sigma^{2}\left\{\frac{A-B}{4 \omega^{2}}\left(1-e^{-2 \alpha^{\prime} \tau}\right)+\frac{(A-B) \alpha}{4 A\left(\alpha^{\prime 2}+\omega^{2}\right)}\right. \\
& \times\left(\frac{A \alpha}{2}-\alpha^{\prime} \rho-\omega\right)+e^{-2 \alpha^{\prime} \tau} \frac{(A-B) \alpha}{4 A\left(\alpha^{\prime 2}+\omega^{2}\right)} \\
& \left.\times\left[(1-\rho) F_{s}(\tau)-\left(1-\frac{\alpha(A-B)}{2 \omega}\right) F_{c}(\tau)\right]\right\},
\end{aligned}
$$

where

$$
\begin{gathered}
F_{s}(\tau)=\omega \sin 2 \omega \tau-\alpha^{\prime} \cos 2 \omega \tau, \\
F_{c}(\tau)=-\left(\omega \cos 2 \omega \tau+\alpha^{\prime} \sin 2 \omega \tau\right) .
\end{gathered}
$$

Note that in a general case there is no simple relation which makes the system dynamics look similar to a simple Brownian one. These expressions have various aspects. First, they contain oscillating functions which are there due to the fact the precession cone is asymmetric. These oscillations disappear for $\tau \rightarrow \infty$ and the final equilibrium solution is independent on the value of damping. The latter is consistent with the fact that in the equilibrium the system statistics should correspond to the Boltzmann distribution.

In two limiting cases the expressions take on a very simple form.

(1) Strong anisotropy. The first case fulfills the conditions $A-B \ll A, B$. This is a rather general case, since the equations imply $\sin ^{2} \theta_{0} \ll 1$ which is easily satisfied in the case of a relatively strong anisotropy. In this case we also have $\rho \ll 1$ and finally the expressions become

$$
\left\langle\left(m_{x}-m_{x}^{0}\right)^{2}\right\rangle_{\text {therm }}=\left\langle\left(m_{y}-m_{y}^{0}\right)^{2}\right\rangle_{\text {therm }}=\frac{\sigma^{2}}{2 \alpha^{\prime}}\left(1-e^{-2 \alpha^{\prime} \tau}\right) \text {. }
$$

As for the spatial correlations, $\left\langle m_{x} m_{y}\right\rangle=\mathcal{O}(A-B) \ll\left\langle\left(m_{x}\right.\right.$ $\left.\left.-m_{x}^{0}\right)\right\rangle_{\text {therm }}^{2}$, and can be neglected. For a time step which fulfills $2 \alpha^{\prime} \tau \ll 1$ we obtain in this case a simple Brownian dynamics solution

$$
\left\langle\left(m_{x}-m_{x}^{0}\right)^{2}\right\rangle_{\text {therm }}=\left\langle\left(m_{y}-m_{y}^{0}\right)^{2}\right\rangle_{\text {therm }}=\sigma^{2} \tau,
$$

which is the formula used previously in the TQMC implementation. ${ }^{9,10}$ It is exactly the solution corresponding to a simple random walk. Note also that this solution could not be obtained if one removes the precessional term from the consideration.

(2) Small damping. The other case which admits simplification is $\alpha \ll 1\left(\alpha^{\prime} \ll \omega\right)$. Here we obtain

$$
\begin{aligned}
& \left\langle\left(m_{x}-m_{x}^{0}\right)^{2}\right\rangle_{\text {therm }}=\frac{\sigma^{2}}{2 \alpha B}\left(1-e^{-2 \alpha^{\prime} \tau}\right), \\
& \left\langle\left(m_{y}-m_{y}^{0}\right)^{2}\right\rangle_{\text {therm }}=\frac{\sigma^{2}}{2 \alpha A}\left(1-e^{-2 \alpha^{\prime} \tau}\right)
\end{aligned}
$$

so that for $\tau \rightarrow \infty$

$$
\frac{\left\langle\left(m_{x}-m_{x}^{0}\right)^{2}\right\rangle_{\text {therm }}}{\left\langle\left(m_{y}-m_{y}^{0}\right)^{2}\right\rangle_{\text {therm }}}=\frac{A}{B}
$$

This limiting value was checked by direct numerical simulation using the Landau-Lifshitz-Gilbert equation with thermal fluctuations introduced as a random field and a good agreement with the analytical prediction was obtained. For short times $\tau$ such that $2 \alpha^{\prime} \tau \ll 1$ it is

$$
\begin{aligned}
& \left\langle\left(m_{x}-m_{x}^{0}\right)^{2}\right\rangle_{\text {therm }}=\sigma^{2} \frac{A+B}{2 B} \tau, \\
& \left\langle\left(m_{y}-m_{y}^{0}\right)^{2}\right\rangle_{\text {therm }}=\sigma^{2} \frac{A+B}{2 A} \tau .
\end{aligned}
$$


The correlations between different components in this case are of the order of $\alpha$ and in principle could be neglected.

However, the strong anisotropy or small damping is not enough for a magnetic particle dynamics to be described by TQMC. Indeed, in order that a formula of the type of Eq. (2.29) can be used for TQMC, and the particle motion could be viewed as a simple random walk, the thermal term should be larger than the a-thermal term so that the latter can be neglected. This leads to the condition

$$
B m_{x}^{0} \tau, \quad A m_{y}^{0} \tau \ll \sigma^{2},
$$

which is fulfilled if either $\alpha$ is large or, alternatively, the temperature is large enough to destroy the influence of the precession. This condition we designate later as corresponding to a diffusion dominated motion. It also requires that the time step $\tau$ can not be chosen too large.

\section{Nonprecessional case}

In the case of real eigenvalues the general solutions of the homogeneous stochastic differential equations can be found in a similar way as for the precessional case using the trial functions

$$
\begin{aligned}
& m_{x}=C_{1} e^{-\lambda_{1} \tau}+C_{2} e^{-\lambda_{2} \tau}, \\
& m_{y}=\widetilde{C}_{1} e^{-\lambda_{1} \tau}+\widetilde{C}_{2} e^{-\lambda_{2} \tau},
\end{aligned}
$$

where $\lambda_{1}=\alpha(A+B) / 2+\omega$ and $\lambda_{2}=\alpha(A+B) / 2-\omega$. To this solutions we add the particular solutions of the inhomogeneous equations. Putting the coefficients $C_{1,2}$ and $\widetilde{C}_{1,2}$ in Eqs. (2.36) and (2.37) as functions of time and substituting in Eqs. (2.14) and (2.15) we find a solution of the stochastic inhomogeneous equation

$$
\begin{aligned}
& C_{1}(\tau)=\frac{\alpha B-\lambda_{2}}{\lambda_{2}-\lambda_{1}} \int_{\tau_{0}}^{\tau} f_{x}(s) e^{\lambda_{1} s} d s+\frac{A}{\lambda_{2}-\lambda_{1}} \int_{\tau_{0}}^{\tau} f_{y}(s) e^{\lambda_{2} s} d s, \\
& C_{2}(\tau)=\frac{\alpha B-\lambda_{1}}{\lambda_{2}-\lambda_{1}} \int_{\tau_{0}}^{\tau} f_{x}(s) e^{\lambda_{1} s} d s+\frac{A}{\lambda_{2}-\lambda_{1}} \int_{\tau_{0}}^{\tau} f_{y}(s) e^{\lambda_{2} s} d s .
\end{aligned}
$$

Hence, the final expression for $\left\langle m_{x}^{2}\right\rangle$ is

$$
\begin{aligned}
\left\langle m_{x}^{2}\right\rangle= & \sigma^{2}\left\{\frac{(G-\omega / A)^{2}+1}{2 \lambda_{1}}\left(1-e^{-2 \lambda_{1} \tau}\right)\right. \\
& +\frac{[G+\omega / A]^{2}+1}{2 \lambda_{2}}\left(1-e^{-2 \lambda_{2} \tau}\right) \\
& \left.-\frac{2\left[G^{2}-\omega^{2} / A^{2}+1\right]}{\left(\lambda_{1}+\lambda_{2}\right)}\left(1-e^{-\left(\lambda_{1}+\lambda_{2}\right) \tau}\right)\right\},
\end{aligned}
$$

and for the correlations:

$$
\begin{aligned}
\left\langle m_{x} m_{y}\right\rangle= & \sigma^{2}\left\{\frac{(G+\omega / A)\left[(G-\omega / A)^{2}+1\right]}{2 \lambda_{1}}\left(1-e^{-2 \lambda_{1} \tau}\right)\right. \\
& +\frac{(G-\omega / A)\left[(G+\omega / A)^{2}+1\right]}{2 \lambda_{2}}\left(1-e^{-2 \lambda_{2} \tau}\right) \\
& \left.-\frac{2 G\left[G^{2}-\omega^{2} / A^{2}+1\right]}{\left(\lambda_{1}+\lambda_{2}\right)}\left(1-e^{-\left(\lambda_{1}+\lambda_{2}\right) \tau}\right)\right\},
\end{aligned}
$$

where $G=\alpha(B-A) / 2 A$. For the limiting solution for $\tau$ $\rightarrow \infty$ we get

$$
\begin{aligned}
& \left\langle m_{x}^{2}\right\rangle_{\text {therm }}=\frac{\sigma^{2}}{2 \alpha B}, \\
& \left\langle m_{y}^{2}\right\rangle_{\text {therm }}=\frac{\sigma^{2}}{2 \alpha A},
\end{aligned}
$$

consistent again with the Boltzmann distribution. Note that this is exactly the same expression as in the case of small $\alpha$, as was also illustrated in Fig. 2. The correlation function

$$
\left\langle m_{x} m_{y}\right\rangle_{\text {therm }}(\tau \rightarrow \infty) \simeq \frac{\sigma^{2} \alpha|B-A|}{2 A}
$$

takes on a finite value for large times while the temporal correlation function $\left\langle m_{x}(\tau) m_{y}(\tau+\Delta \tau)\right\rangle$ goes to zero for $\Delta \tau \rightarrow \infty$.

For small times $\tau \ll 1 / \lambda_{1}, 1 / \lambda_{2}$ we obtain the normal diffusion coefficient

$$
\left\langle m_{x}^{2}\right\rangle_{\text {therm }}=\left\langle m_{x}^{2}\right\rangle_{\text {therm }}=\sigma^{2} \tau .
$$

Note that in the same approximation there is no contribution from the athermal part. The latter means that in the case of large damping there could be a complete analogy between the Brownian particle and magnetic particle motions. However, the correlational part in this case is not small:

$$
\left\langle m_{x} m_{y}\right\rangle=\frac{2 \alpha \sigma^{2}\left(B^{2}-A^{2}\right)}{A^{2}} \tau .
$$

Since neither $\alpha$ or $B-A$ are small in this case, the correlations are of the order of $\left\langle m_{x}^{2}\right\rangle$.

\section{IMPLEMENTATION OF THE TIME QUANTIFIED MONTE CARLO METHOD}

In this paper we will consider only implementation based on the use of the simplest diffusion coefficient of the form of Eq. (2.29). Other possibilities are currently under investigation and will be published elsewhere.

Within a MC algorithm, it is convenient to make a trial step move in a certain cone of radius $R$. In a simple case where the diffusion coefficient $D$ is defined by properties of the fluctuating forces [see Eq. (2.29)], we will compare the fluctuations which are established in the MC algorithm with the fluctuations within a given time scale associated with the linearized stochastic LLG equation.

For this comparison, first we calculate the fluctuations 
$\left\langle M_{x}^{2}\right\rangle$ which are established within one step of the MC simulation. The trial step of our MC implementation is a random movement of the magnetic moment up to a certain maximum opening angle (inside the cone of the radius $R$ around the current magnetization position). In order to achieve this efficiently we first construct a random vector with constant probability distribution within a sphere of radius $R$ by use of the rejection method. ${ }^{24}$ This random vector is then added to the initial moment and subsequently the resulting vector is again normalized. Note that the probability distribution for trial steps of size $r=\sqrt{M_{x}^{2}+M_{y}^{2}}$ is $p_{t}=3 \sqrt{R^{2}-r^{2}} /\left(2 \pi R^{3}\right)$ for $0<r<R$. It is nonuniform but isotropic, so that the symmetry condition is fulfilled. The acceptance probability using a heat-bath algorithm is given by

$$
w_{s \rightarrow s^{\prime}}=\frac{w_{0}}{1+\exp \left(\frac{E\left(S^{\prime}\right)-E(S)}{k_{B} T}\right)},
$$

where $S$ and $S^{\prime}$ denote two different states of the system and $w_{0}$ is a constant.

Assuming that the spin is close to its (local) equilibrium position, $\Delta E\left(r^{2}\right)$ from Eq. (2.6) can be expanded for small $r$, yielding

$$
\Delta E\left(r^{2}\right)=K V\left(1+2 H_{z}\right) r^{2}
$$

for the symmetric case where the field is parallel to the easy axis. In order to calculate the fluctuations within one MC step (MCS) we have to integrate over that part of the phase space which can be reached within one MCS,

$$
\begin{aligned}
\left\langle M_{x}^{2}\right\rangle & =\int_{0}^{2 \pi} \mathrm{d} \varphi \int_{0}^{R} r \mathrm{~d} r \frac{r^{2}}{2} w(r) p_{t}(r) \\
& =\frac{R^{2}}{10}-\frac{K V\left(1+2 H_{z}\right) R^{4}}{k_{B} T}+\mathcal{O}\left(R^{4}\right),
\end{aligned}
$$

where the last line is an expansion for small $R$. The second term can be dropped for sufficiently small $R$, leading to the condition

$$
R^{2} \ll k_{B} T / K V\left(1+2 H_{z}\right) .
$$

By equalizing the fluctuations within a time interval $\Delta t$ of the LLG equation and one MCS we find the relation

$$
R^{2}=\frac{10 k_{B} T \alpha}{K V} \Delta \tau=\frac{20 k_{B} T \alpha \gamma}{\left(1+\alpha^{2}\right) \mu_{s}} \Delta t
$$

for the trial step width $R .{ }^{9}$ Equation (3.5) is the central result of TQMC. It relates one MC step, performed using an algorithm as explained before, with a real time interval of the solution of the Langevin equation. Corresponding relations for other trial step distributions or other acceptance probabilities, as for instance following from a Metropolis algorithm, can be derived as well. Also, in the same way the time step quantifications could, in principle, be taken from Eq. (2.28) or Eqs. (2.33) and (2.34).
Note, that from the derivation above it follows that one time step $\Delta t$ must be larger than the intrinsic time scale of the relaxation. This means that results from the MC method can only be interpreted on time scales that are clearly larger than the microscopic time scale of the (local) relaxation of the spin.

The most interesting result of Eq. (3.5) is the temperature (or barrier) dependence on the trial radius $R$. If the barrier is large, in theory this allows the introduction of a larger time step $\Delta \tau$ as in the integration of the Landau-Lifshitz equation.

In principle, Eq. (3.5) allows the possibility to choose the trial step width for a MC simulation in such a way that $1 \mathrm{MC}$ step corresponds to some microscopic time interval, say $\Delta t$ $=10^{-12} \mathrm{~s}$. However, there are of course restrictions for possible values of the trial step width: $R$ must be small enough so that the truncated expansion in Eq. (3.3) is a good approximation. On the other hand $R$ should not be too small since otherwise the MC algorithm needs too much computation time to sample the phase space. Therefore, either one has to choose such a value for $\Delta t$ so that $R$ takes on reasonable values, or one chooses a reasonable constant value for $R$ and uses Eq. (3.5) to calculate $\Delta t$ as the real time interval associated with the MC cycle. In the following we will use the first method since it turns out to be very efficient to change $R$ with temperature. Also, in this case it is much easier to control the fulfillment of condition (3.4). However, the alternative method yields the same results ${ }^{10}$ as long as condition (3.4) is not violated.

\section{COMPARISON OF THE TWO DYNAMICS AND DISCUSSION}

In this section we compare the TQMC method and the Langevin dynamics calculating the switching time of a magnetic particle. This time is defined as an averaged (over many realizations) time necessary for the particle to change the direction of the magnetization, i.e., until the $M_{z}$ component changes its sign. Note that the switching time essentially is a nonequilibrium quantity.

First of all we should clarify the conditions under which the TQMC method was used previously basing on the analysis presented in this paper. Most of the previously considered cases $^{9,11}$ were under the conditions of the precessional regime where it is $\alpha<\alpha_{\text {cr }}$ [see Eq. (2.17)]. The parameters corresponding to Fig. 4 of the original TQMC paper $^{9}$ give the values $A=0.877$ and $B=0.781$, yielding a very large value of $\alpha_{\mathrm{cr}} \approx 17$. Similar considerations are true for most of the magnetic recording applications ${ }^{11}$ since even if the local grain anisotropy is perpendicular to the applied field value, its value is normally sufficiently strong to assure that the condition $|A-B| \ll A, B$ is fulfilled. This means that calculations usually are made under the conditions of the precessional motion where Eq. (2.29) is valid and the TQMC method must work in the high damping limit.

For a smaller damping one expects the precession itself to contribute significantly to the reversal process. The elliptical motion of the athermal part of Eqs. (2.20) and (2.21) can increase the distance of the magnetic moment from its equilibrium position: an initial deviation $m_{y}^{0}$ of the moment along 


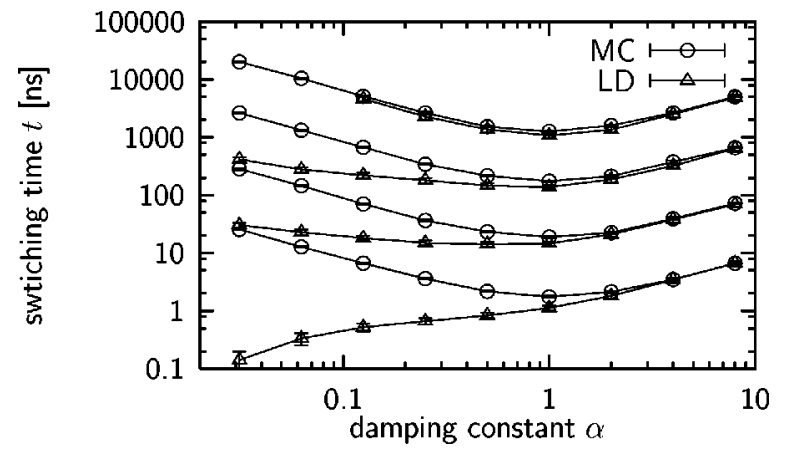

FIG. 3. Average switching time versus damping constant for a magnetic moment in a field $H / 2 K V=0.42$ applied under an angle of $0, \pi / 20, \pi / 10 /, \pi / 4$ to the easy axis (from top). Comparison between Langevin dynamics (LD) and time-quantified Monte Carlo (MC) $2 K V / k_{B} T=72$.

the hard axis (in the following the $y$ axis with $A \geqslant B$ ) at time $\tau=0$ leads after a quarter of the precession time to a deviation from the equilibrium position along the easy $x$ axis which can be larger than the original distance and, hence contributes to the escape from the local equilibrium position. Hence, the influence of precession can only be neglected when the distance does not grow due to the precession. This leads to the condition

$$
S_{x}(\tau=\pi / 2 \omega)=S_{y}^{0} \frac{A}{\omega} e^{-\pi \alpha^{\prime} / 2 \omega} \ll S_{y}^{0},
$$

from which follows a condition for the damping constant

$$
\alpha \gg \frac{4 \omega}{\pi(A+B)} \ln (A / \omega) .
$$

In the limit of small $\alpha$ the latter simplifies to

$$
\alpha \gg \frac{2 \sqrt{A / B}}{\pi(1+A / B)} \ln (A / B) .
$$

The implication of the condition above is illustrated in Fig. 3 where results for the switching time of a magnetic moment in a field with different angles to the easy axis stemming from TQMC and Langevin dynamics simulations, respectively, are compared. In the symmetric case $(A / B=1)$ the condition above is alway fulfilled so that the TQMC method works for all values of $\alpha$. The more asymmetric the problem is, the bigger becomes that value of $\alpha$ above which the TQMC method works correctly. Note that if one is interested in the long time behavior, for small driving fields, where the energy barriers are large the TQMC method will work even better since here it is usually $A \approx B$.

The influence of precession can also be neglected when the thermal fluctuations are large enough to destroy the precessional motion. This requires the condition (2.35) to be satisfied. Figure 4 illustrates this condition where we present the switching time as a function of the damping parameter for various temperatures. If the temperature is fixed there will always exist a critical value $\alpha_{\text {cr }}$ of the damping parameter where the condition (2.35) is violated. Consequently, if

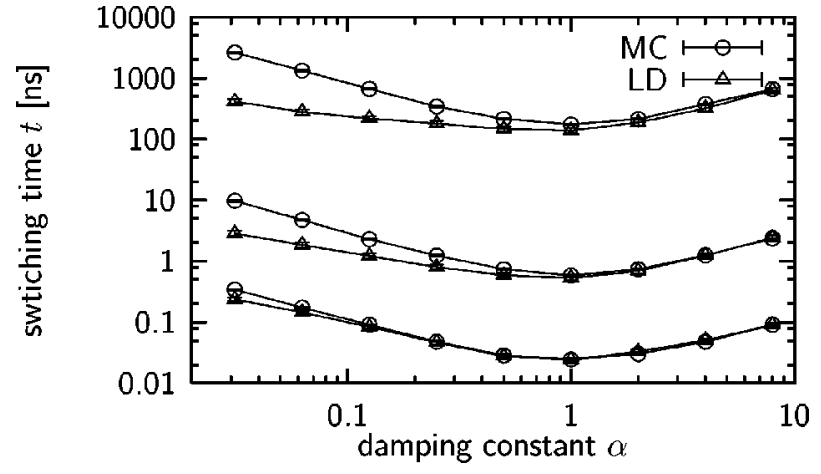

FIG. 4. Average switching time versus damping constant for a magnetic moment in a field $H / 2 K V=0.42$ applied under an angle of $\pi / 20$ to the easy axis for different temperatures $2 \mathrm{KV} / \mathrm{k}_{B} T$ $=72,7.2,2.1$ (from top).

we work in the small damping regime, the temperature must be large enough to suppress the influence of the elliptical precessional motion.

In principle, since the time quantification is not restricted to the use of Eq. (2.33), some other ideas of how to include the precessional term into the diffusional coefficient may appear in future. In our implementation of TQMC the axially symmetric case is the only one where the method works for all the damping parameter values. This is once again demonstrated in Fig. 5. Also shown in Fig. 5 is that the MC approach correctly describes the Langevin dynamics even for small barriers when the Fokker-Planck asymptote fails.

Similar results are presented in Fig. 6 for the nonprecessional case where the condition $A-B \ll A, B$ is not fulfilled. To model clearly this case we suppose that there is a hard axis anisotropy in $y$ direction in addition to easy axis in the $z$ direction. In this case the values of $A$ and $B$ can be very different since it is

$$
A=1+H_{\mathrm{appl}}
$$

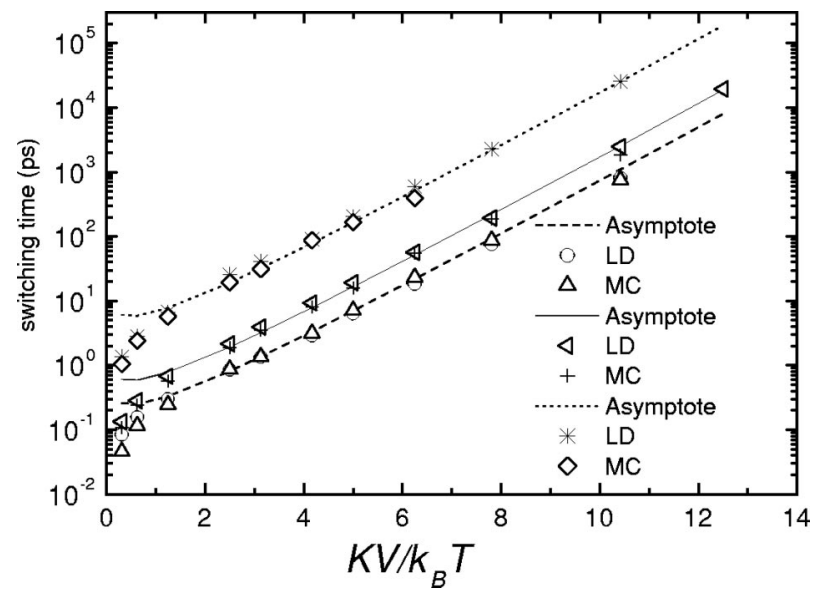

FIG. 5. Average switching time as a function of the energy barrier for various damping constant $\alpha=4,0.1,0.01$ (from top) in an axially symmetric case. Comparison between Langevin dynamics (LD), time-quantified Monte Carlo (MC), and Brown's asymptote (Ref. 5). 


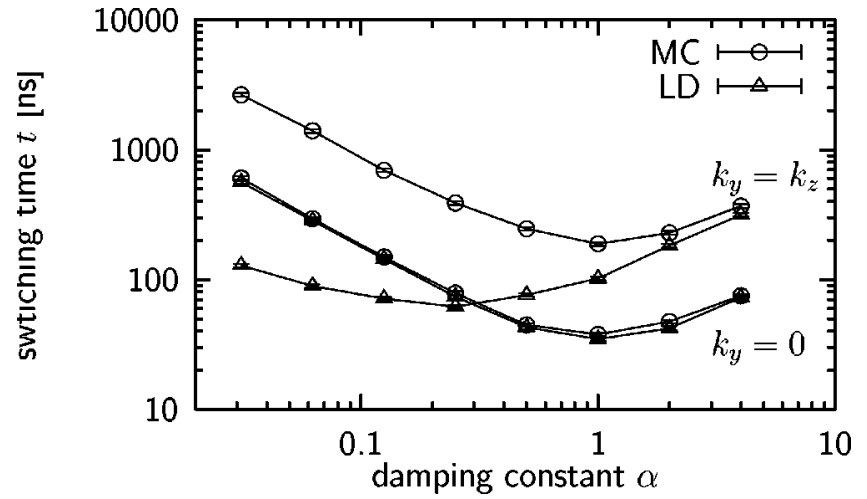

FIG. 6. Average switching time versus damping constant for a magnetic moment with an easy axis anisotropy $2 K_{z} V / k_{B} T=100$ and different additional hard axis anisotropies. The applied field $H / 2 K_{z} V=0.9$ is parallel to the easy axis.

and

$$
B=1+d_{y}+H_{\mathrm{appl}} .
$$

Here $d_{y}=K_{y} / K_{z}$ and $\alpha_{\text {cr }}$ can take on any value depending on the value of $K_{y}$. In Fig. 6 we present the escape time as a function of the damping parameter for several values of the parameter $A-B$ defined by the perpendicular anisotropy $d_{y}$. Note that the method fails if the value of $d_{y}$ is increased which we attribute to the fact that the correlations (not taken into account in the present implementation) become important according to Eq. (2.46).

Although the correct magnetization values were calculated for a noninteracting system TQMC, in the implementation presented above, has been successfully applied of interacting systems as well. ${ }^{25,26}$ As an example to that, in Fig. 7 we present the switching time as a function of the exchange constant in a linear chain of magnetic particles with easy anisotropy axis parallel to the field direction. As the nearest neighbor exchange parameter $J$ is increased, the system undergoes first a transition from isolated magnetic moments to a nucleation-propagation mechanism and for still larger values of $J$ to a magnetization reversal by coherent rotation (see also Ref. 25). At this point, the switching time becomes independent on the exchange constant. It is interesting, that the

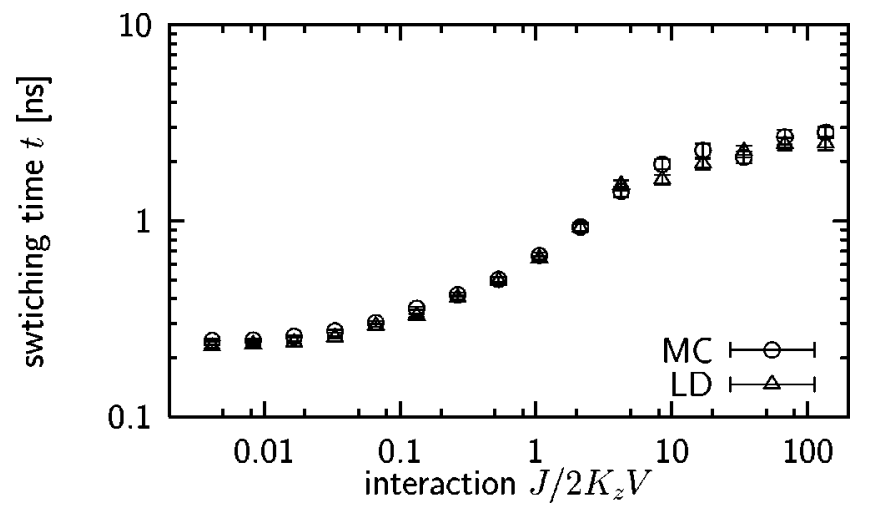

FIG. 7. Average switching time versus strength of the nearest neighbor interaction in a chain of 16 moments. $2 K V / k_{B} T=72, \alpha$ $=0.1$. The field $H / 2 K V=0.95$ is parallel to the easy axis.

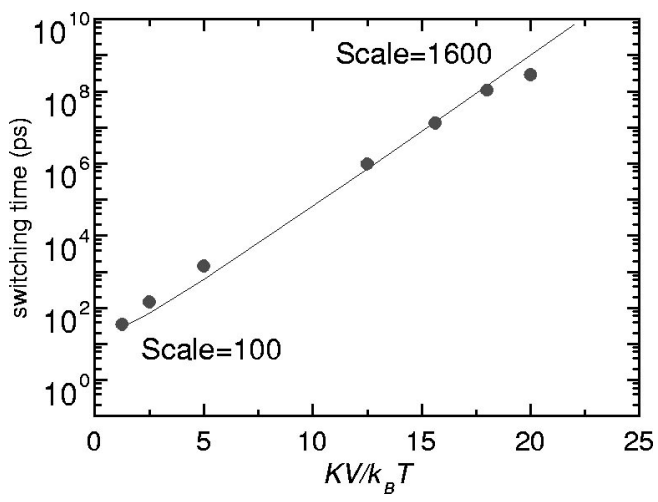

FIG. 8. Average switching time vs energy barrier calculated with TQMC compared with Aharoni's asymptote (Ref. 6) for the axially symmetric case. During the simulation the trial MC step size $R=0.111$ was kept constant. The corresponding quantified time step $\Delta t_{\mathrm{MC}}$ was increased proportionally to the barrier size $\Delta t_{\mathrm{MC}}$ $=\mathrm{Scale} \times \Delta t_{\mathrm{LL}}$ where $\Delta t_{\mathrm{LL}}=0.01$ of the precession period is the normal Landau-Lifshitz integration time step. The parameters of the simulation $\alpha=0.1, H_{\text {app }}=0.15$.

TQMC was able to correctly describe the predicted behavior. However, one could expect the appearance of magnetization correlations due to the dynamic coupling, which should be taken into account in an improved MC time quantification scheme for interacting systems. An attempt to introduce such correlations was taken in Refs. 27,28. Interestingly, even without such correlations the TQMC correctly reproduces the Langevin dynamics results for relatively large damping values.

Finally, we should point out that since $R^{2}$ in Eq. (3.5) is proportional to the time step and inversely proportional to the barrier height $K V / k_{B} T$, this allows one to use a big time step for big barrier height, always when the condition (3.4) is fulfilled. In Fig. 8 we present the switching time as a function of the barrier height. In this case the MC trial step was kept constant to 0.111 and the time step was progressively increased with the increment of the barrier height. The deviations for the large barrier may be attributed to the fact that finally the condition (3.4) fails. In the same figure we present for comparison Aharoni's asymptote ${ }^{6}$ for the magnetization reversal and note a good agreement between the approaches.

\section{CONCLUSIONS AND FUTURE WORK}

Since Langevin dynamics is useful for investigating only fast relaxation processes, studies of thermal stability require the development of more computationally efficient methods. For intermediate time scales it is desirable to work with methods based on MC algorithms but incorporating a time quantification. At the moment we have found no unique way to implement TQMC in the general case. In the present paper we report only the simplest case of the diffusional coefficient based on formula (2.29). We have found that this implementation for TQMC should work provided several conditions.

(1) Diffusion dominated motion. The pure precessional motion should be small compared to the diffusion coefficient. The latter could be fulfilled either for high temperature or large damping value. 
(2) Uncorrelated motion. The spatial correlations between magnetic moment components should be small compared to the diffusion coefficient.

In practice, we have found that the application of the method is reasonable in a system of relatively large easy anisotropy and intermediate-to-hight damping value. The close to axially symmetric case is a special case when the MC procedure gives perfectly the same answer as the dynamical equation integration. This is explained, probably, by the fact that in this case the energy barrier is the same in all the directions and, consequently, it does not matter for the particle at which point of the space to cross it. However, in a nonaxially symmetric case there exist only two points in space where the barrier is lowered and, consequently, the particle would go preferably through them. The dynamical precession helps the particle to explore more directions of the space, takes the system closer to the transition point and, then the noise helps to overcome the barrier. Thus, the dynamical precession plays a significant role in the switching process and reduces the switching time in comparison to a pure random walk.

In principle, the use of TQMC is not restricted to formula (2.29) and implementation of the current paper. In the future, other implementations based on different diffusion coefficient estimations which includes precession or TQMC with correlations may appear. This work is currently in progress.

We have found some evidence that the simple formulas apply also to the case of interacting moments, although this needs further investigation. Future work will involve an in- vestigation of interacting particle systems, along with the development of methods suitable for systems undergoing precession dominated motion.

The method rests on a comparison with Langevin dynamics. Here, the coupling to the heat bath is added phenomenologically to the equation of motion leading to a damping constant $\alpha$, the microscopic evaluation of which is still missing. In this sense there is still a lack of an absolute microscopic time scale. Nevertheless, there is at least a nontrivial connection between MC methods and Langevin dynamics. In this sense, our results also show that MC methods are viable to describe switching processes in magnetic systems and to give realistic dynamics. Finally and importantly, Metropolis Monte Carlo with quantified time step constitutes a numerical method which is much faster than the integration of the dynamical equation of motion.

\section{ACKNOWLEDGMENTS}

O.C. acknowledges the hospitality and support from Durham University, U.K., Duisburg University, Germany, and Seagate Research Center, Pittsburgh, where a part of this work was done. R.S.-R. thanks Durham University, U.K., for hospitality and support. M.A.W. thanks ICMM, Madrid, Spain for hospitality and support, and acknowledges the EPSRC who supported this project under Grant No. R040 318.
${ }^{1}$ W. F. Brown, Phys. Rev. 130, 1677 (1963).

${ }^{2}$ D. T. Gillespie, J. Comput. Phys. 22, 403 (1976).

${ }^{3}$ Y. Kanai and S. H. Charap, IEEE Trans. Magn. 27, 4972 (1991).

${ }^{4}$ Y. Zhang and H. N. Bertram, IEEE Trans. Magn. 34, 3786 (1998).

${ }^{5}$ W. F. Brown, IEEE Trans. Magn. MAG-15 (1979).

${ }^{6}$ A. Aharoni, Phys. Rev. 177, 793 (1969).

${ }^{7}$ H. B. Braun, Phys. Rev. Lett. 71, 3557 (1993).

${ }^{8}$ W. T. Coffey, D. S. F. Crothers, J. L. Dormann, L. J. Geoghan, and E. C. Kennedy, Phys. Rev. B 58, 3249 (1998).

${ }^{9}$ U. Nowak, R. W. Chantrell, and E. C. Kennedy, Phys. Rev. Lett. 84, 163 (2000).

${ }^{10}$ R. Smirnov-Rueda, O. Chubykalo, U. Nowak, R. W. Chantrell, and J. M. Gonzalez, J. Appl. Phys. 87, 4798 (2000).

${ }^{11}$ O. A. Chubykalo, J. Kaufman, B. Lengsfield, and R. SmirnovRueda, J. Magn. Magn. Mater. 242-245 (2002).

${ }^{12}$ D. Stauffer, F. W. Hehl, V. Winkelmann, and J. G. Zabolitzky, Computer Simulation and Computer Algebra (Springer-Verlag, Berlin, 1993).

${ }^{13} \mathrm{~K}$. Binder and D. W. Heermann, in Monte Carlo Simulation in Statistical Physics, edited by P. Fulde (Springer-Verlag, Berlin, 1997).

${ }^{14}$ R. Kubo, M. Toda, and N. Hashitsume, Statistical Physics II: Nonequilibrium Statistical Mechanics (Springer-Verlag, Berlin, 1985).

${ }^{15}$ F. Reif, Fundamentals of Statistical and Thermal Physics (McGraw-Hill, New York, 1967).
${ }^{16}$ K. Kikuchi, M. Yosida, T. Mackawa, and H. Watanabi, Chem. Phys. Lett. 185, 335 (1991).

${ }^{17}$ E. Meiburg, Phys. Fluids 29, 3107 (1986).

${ }^{18}$ J. M. Gonzalez, R. Ramirez, R. Smirnov-Rueda, and J. Gonzalez, Phys. Rev. B 52, 16034 (1995).

${ }^{19}$ J. M. Gonzalez, O. A. Chubykalo, and R. Smirnov-Rueda, J. Magn. Magn. Mater. 203, 18 (1999).

${ }^{20}$ O. A. Chubykalo, J. M. Gonzalez, G. R. Aranda, and J. Gonzalez, J. Magn. Magn. Mater. 35, 314 (2000).

${ }^{21}$ R. Smirnov-Rueda, O. A. Chubykalo, J. M. Gonzalez, and J. Gonzalez, J. Appl. Phys. 83, 6509 (1998).

${ }^{22}$ J. L. Garcia-Palacios and F.-J. Lázaro, Phys. Rev. B 58, 14937 (1998).

${ }^{23}$ A. Lyberatos, D. V. Berkov, and R. W. Chantrell, J. Phys.: Condens. Matter 5, 8911 (1993).

${ }^{24}$ F. Vesely, Computational Physics (Universitätsverlag, Wien, 1993).

${ }^{25}$ D. Hinzke and U. Nowak, Phys. Rev. B 61, 6734 (2000).

${ }^{26}$ D. Hinzke and U. Nowak, J. Magn. Magn. Mater. 221, 365 (2000).

${ }^{27}$ R. Smirnov-Rueda, J. D. Hannay, O. Chubykalo, R. W. Chantrell, and J. M. González, IEEE Trans. Magn. 35, 3730 (1999).

${ }^{28}$ O. A. Chubykalo, B. Lengsfield, B. Jones, J. Kaufman, J. M. Gonzalez, R. W. Chantrell, and R. Smirnov-Rueda, J. Magn. Magn. Mater. 221, 132 (2000). 